\title{
Discrete-time detection modeling for unsaturated ocean acoustic propagation
}

\author{
Anastassios N. Perakis \\ Department of Naval Architecture and Marine Engineering, The University of Michigan, Ann Arbor, \\ Michigan 48109 \\ Harilaos N. Psaraftis \\ Department of Ocean Engineering, Massachusetts Institute of Technoloty, Cambridge, Massachusetts 02139
}

(Received 21 March 1983; accepted for publication 3 August 1983)

The basic problem in ocean acoustic detection is formulated under the assumption of unsaturated sound propagation. The latter essentially amounts to a constant signal plus Gaussian noise. Detection is defined as occurring whenever $\rho$, the root mean square pressure at the receiver, exceeds a specified threshold level $\rho_{0}$. A two-state, discrete-time Markov model is derived, and closed-form expressions for the probability mass functions of the number of time steps separating two successive detections (interarrival time) or one detection and the first subsequent "downcrossing" (holding time) are presented. Expressions for the joint probability density function of $\rho$ at two different points in time are obtained and used to determine the relevant onestep transition probabilities of the Markov model. Sample results using the model are finally presented.

PACS numbers: $43.60 . \mathrm{Cg}, 43.30 . \mathrm{Bp}, 92.10 . \mathrm{Vz}$

\section{INTRODUCTION}

In general, the quadrature components of the envelope of a narrow-band ocean acoustic multipath process are given by $^{1}$

$$
\begin{aligned}
& X=\sum_{n=1}^{N}\left(r_{n} \cos \theta_{n}+N_{x}^{(n)}\right), \\
& Y=\sum_{n=1}^{N}\left(r_{n} \sin \theta_{n}+N_{y}^{(n)}\right),
\end{aligned}
$$

where

$N=$ number of independent paths between source and receiver,

$r_{n}=$ the amplitude of the $n$th path,

$\theta_{n}=$ the phase of the $n$th path

$N_{x}^{(n)}, . N_{y}^{(n)}=$ zero-mean, uncorrelated Gaussian additive noise for the $n$th path.

Furthermore, the envelope and the phase of the total signal are defined as:

$$
\begin{aligned}
& \rho=\left(X^{2}+Y^{2}\right)^{1 / 2}, \\
& \phi=\tan ^{-1}(Y / X) .
\end{aligned}
$$

At short ranges and low frequencies, or for stable channels, the propagation is said to be unsaturated and the probability density function (PDF) of $\rho$ is Rician ${ }^{2}$ and independent of the number of paths.' (In Sec. I the distributions of $\rho$ and its phase $\phi$ are presented.)

At sufficiently long ranges and/or high frequencies, the propagation is fully saturated, which means that $\phi$, the phase of $\rho$, can be characterized as a random variable uniformly distributed between 0 and $2 \pi$, or each path has a phase $\theta_{n}$ that is normally distributed with a standard deviation $>2 \pi$. In this regime when $N>4$ and the single path amplitudes $r_{n}$ are approximately equal, phase random multipath propaga- tion is obtained. It has been found $d^{3-5}$ that the envelope $\rho$ of a fully saturated phase random process with additive Gaussian noise obeys a Rayleigh PDF. Moreover, several other statistics and joint PDF's for the phase random process have been obtained, and are presented in a comprehensive summary by Mikhalevsky. ${ }^{6}$

In intermediate ranges, where the signal experiences enough perturbations in the channel so that each $\theta_{n}$ can be characterized as a Gaussian random variable but with a standard deviation $<2 \pi$, partially saturated propagation is obtained. The frequency/range boundaries between the unsaturated, partially saturated, and fully saturated regimes are dependent upon the ocean dynamics or boundary dynamics of the propagation channel, as well as the magnitude of any relative source-receiver motion. In Ref. 1 , the envelope statistics for signals in the partially saturated regime were presented. As the variance of the single path phase goes to zero, or becomes large, the PDF's converge to the unsaturated and fully saturated results, respectively. ${ }^{12}$

In previous publications ${ }^{7.8}$ of the authors, continuous and discrete-time detection models using the results of phase random acoustic propagation ${ }^{3-6}$ have been formulated. "Detection" was defined as an upcrossing of random variable $\rho$ (the root mean square pressure at the passive sonar receiver) over a specified threshold $\rho_{0}$. A continuous-time model was first developed for obtaining the PDF's of the time between a detection and the first subsequent downcrossing through $\rho_{0}$ (holding time). The model was then compared with the extensively used $(\lambda, \sigma)$ model and with available acoustic data. This model was seen to exhibit similar long-term behavior but markedly different short term characteristics as compared with the $(\lambda, \sigma)$ model, a fact which is due to the memory of the process. Comparison with data has demonstrated, in most cases, a significantly improved prediction capability over the $(\lambda, \sigma)$ model. 
Subsequently, a two-state model and a four-state discrete-time Markov detection model were developed, and closed-form expressions for the probability mass functions of the corresponding interarrival and holding times were derived. The results obtained using the latter models were favorably compared with both the continuous-time models and the data, the greatest improvement over the continuoustime models lying in the much lower computational effort involved.

The purpose of this letter is to develop acoustic detection models for the unsaturated case. Such models are derived in the following section, first for the memoryless case and then for the general "memory" Markov case. A model has also been proposed ${ }^{9}$ for the partially saturated case, but results are yet to be confirmed in other than limiting cases.

\section{ANALYTICAL FORMULATION}

The probability density functions for the root mean square pressure $\rho$ and its phase $\phi$ for the unsaturated process are derived in Ref. 1. The density of $\rho$ is Rician:

$f_{\rho}(\rho)=\frac{\rho}{\sigma_{N}^{2}} \exp \left(-\frac{\rho^{2}+R_{S}^{2}}{2 \sigma_{N}^{2}}\right) I_{0}\left(\frac{\rho R_{S}}{\sigma_{N}^{2}}\right), \quad 0<\rho<\infty$,

where

$\boldsymbol{R}_{s}=$ the magnitude of the constant signal vector,

$I_{0}=$ modified Bessel function of the first kind of zero order, $\sigma_{N}^{2}=N \sigma_{N_{x}}^{2}=N \sigma_{N_{y}}^{2}$, where

$\sigma_{N_{x}}^{2}, \sigma_{N_{y}}^{2}=$ the variances of $N_{x}^{(n)}$ and $N_{y}^{(n)}$, respectively, as defined in the introduction.

The density of $\phi$ is

$$
\begin{aligned}
f_{\phi}(\phi)= & \frac{1}{2 \pi \sigma_{N}^{2}} \exp \left(\frac{-R_{S}^{2}}{2 \sigma_{N}^{2}}\right)\left[\sigma_{N}^{2}+\sigma_{N} M_{S}\left(\frac{\pi}{2}\right)^{1 / 2}\right. \\
& \left.\times \exp \left(\frac{M_{S}^{2}}{2 \sigma_{N}^{2}}\right)\left[1-\Phi\left(\frac{M_{S}}{\left(2 \sigma_{N}^{2}\right)^{1 / 2}}\right)\right]\right\},
\end{aligned}
$$

where

$$
M_{s}=\mu_{x} \cos \phi+\mu_{y} \sin \phi,
$$

$\mu_{x}, \mu_{y}=$ the quadrature components of $R_{S}\left(R_{S}^{2}=\mu_{x}^{2}+\mu_{y}^{2}\right)$, and

$$
\Phi(x)=\operatorname{erf}(x)=\frac{2}{\sqrt{\pi}} \int_{0}^{x} \exp \left(-t^{2}\right) d t .
$$

In our previous Markov modeling of the phase random process (fully saturated sound propagation), a two-state model and a four-state model were developed. ${ }^{7-8}$ Comparison with data has revealed that both models, when properly calibrated, yield very satisfactory results, the two-state being consistently as accurate as the four-state model. ${ }^{8}$

We will henceforth restrict ourselves in developing a two-state Markov model for the unsaturated process of the general form shown in Fig. 1,

$$
\begin{gathered}
\text { where " } U "=\text { "up" state, defined by } \rho>\rho_{0}, \\
\text { " } D "=\text { "down" state, defined by } \rho<\rho, \\
a=\operatorname{prob}\left(\rho_{2}<\rho_{0} \mid \rho_{1}>\rho_{0}\right), \\
b=\operatorname{prob}\left(\rho_{1}>\rho_{0} \mid \rho_{2}<\rho_{0}\right) .
\end{gathered}
$$

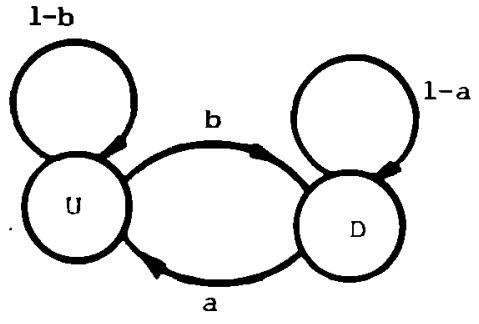

FIG. 1. Two-state discrete-time Markov model.

In the memoryless case, $a+b=1$, and

$$
\begin{aligned}
a & =\operatorname{prob}\left(\rho_{2}<\rho_{0} \mid \rho_{1} \geqslant \rho_{0}\right)=\operatorname{prob}\left(\rho_{2}<\rho_{0} \mid \rho_{1}<\rho_{0}\right) \\
& =\operatorname{prob}\left(\rho_{2}<\rho_{0}\right)
\end{aligned}
$$

or

$$
a=\int_{0}^{\rho_{0}} f_{\rho}(\rho) d p=1-Q_{1}\left(\frac{R_{S}}{\sigma_{N}}, \frac{\rho_{0}}{\sigma_{N}}\right)
$$

with $f_{f}(\rho)$ as in (3), and the generalized $Q$-function is defined ${ }^{10}$ as

$Q_{M}(\alpha, \beta)=\int_{\beta}^{\infty} \xi\left(\frac{\xi}{\alpha}\right)^{M-1} \exp \left(-\frac{\alpha^{2}+\xi^{2}}{2}\right) I_{M-1}(\alpha \xi) d \xi$,

$M=1,2, \ldots$.

It can be shown that

$$
\begin{aligned}
1-Q_{1}(\alpha, \beta)= & \exp \left(-\frac{\alpha^{2}+\beta^{2}}{2}\right) \\
& \times \sum_{k=0}^{\infty} \frac{\left(\alpha^{2} / 2\right)^{k}}{k !} \sum_{n=k+1}^{\infty} \frac{\left(\beta^{2} / 2\right)^{n}}{n !} .
\end{aligned}
$$

Rappaport ${ }^{10}$ presents several approximations for $Q$ and its complement, which is less cumbersome to evaluate exactly in the computer than using (8). Note: Rice $^{2}$ presents the equivalent result:

$a=\int_{0}^{\rho_{a}} f_{\rho}(\rho) d \rho=\exp \left(\frac{\rho_{0}^{2}+R_{S}^{2}}{2 \sigma_{N}^{2}}\right) \sum_{n-1}^{\infty}\left(\frac{\rho_{0}}{R_{S}}\right)^{n} I_{n}\left(\frac{\rho_{0} R_{S}}{\sigma_{N}^{2}}\right)$,

where

$$
I_{n}(x)=\left(\frac{x}{2}\right)^{n} \sum_{k=0}^{\infty}\left(\frac{x}{2}\right)^{2 k} \frac{1}{k !(n+k) !} .
$$

From (9), we can proceed to evaluate the probability mass functions for the interarrival and holding time. In general, these PMF's take the form ${ }^{8}$

$$
\begin{aligned}
& P_{H}(k)=(1-a)^{k-1} a, \quad k=1,2, \ldots \text { (holding time), } \\
& P_{\ell}(n)=[a b /(a-b)]\left[(1-b)^{n-1}-(1-a)^{n-1}\right], \\
& n=2,3, \ldots \text { (interarrival time). }
\end{aligned}
$$

In the memoryless case, (11) becomes

$$
P_{I}(n)=\frac{a(1-a)}{(1-2 a)}\left[(1-a)^{n-1}-a^{n-1}\right], \quad n=2,3, \ldots .
$$

In the nontrivial case $(a+b \neq 1)$ of the Markov model of Fig. 1, the calculation of the transition probabilities requires knowledge of the joint density function $f_{P_{P} p_{1}}\left(\rho_{1}, \rho_{2}\right)$, 
where $\rho_{1}=\rho(t)$ and $\rho_{2}=\rho(t+T)$. This second-orderdensity has already been derived in a rather general form by Middleton's ${ }^{11}$ treatment of the statistical properties of additive narrowband signal and normal noise processes.

Using Middleton's results, and after extensive algebraic manipulations, we obtain

$$
\begin{aligned}
f_{\rho_{1}, \rho_{2}}\left(\rho_{1}, \rho_{2}\right)= & \frac{\rho_{1} \rho_{2}}{\sigma_{N}^{2}\left(1-r_{0}^{2}\right)} \exp \left(-\frac{\rho_{1}^{2}+\rho_{2}^{2}}{2 \sigma_{N}^{2}\left(1-r_{0}^{2}\right)}\right) \\
& \cdot \times \exp \left(\frac{A_{0}^{2}}{\sigma_{N}^{2}\left(1+r_{0}\right)}\right) \\
& \cdot \sum_{m=0}^{\infty} \epsilon_{m} I_{m}\left(\frac{r_{0} \rho_{1} \rho_{2}}{\sigma_{N}^{2}\left(1-r_{0}^{2}\right)}\right) \\
& \times I_{m}\left(-\frac{A_{0} \rho_{1}}{\sigma_{N}^{2}\left(1+r_{0}^{2}\right)}\right) I_{m}\left(\frac{A_{0} \rho_{2}}{\sigma_{N}^{2}\left(1+r_{0}\right)}\right)
\end{aligned}
$$

It is reasonable to expect that (13) will reduce, for $t \rightarrow \infty$, to the product of $f_{\rho_{1}}\left(\rho_{1}\right) \cdot f_{\rho_{2}}\left(\rho_{2}\right)$, i.e.,

$$
\begin{aligned}
\underset{\substack{\rho_{1}, \rho_{2} \\
\rightarrow \rightarrow \infty}}{ }\left(\rho_{1}, \rho_{2}\right) \rightarrow & \frac{\rho_{1} \rho_{2}}{\sigma_{N}^{4}} \exp \left(-\frac{\rho_{1}^{2}+\rho_{2}^{2}+2 A_{0}^{2}}{2 \sigma_{N}^{2}}\right) \\
& \times I_{0}\left(\frac{A_{0} \rho_{1}}{\sigma_{N}^{2}}\right) I_{0}\left(\frac{A_{0} \rho_{2}}{\sigma_{N}^{2}}\right) .
\end{aligned}
$$

Assuming $k_{0} \rightarrow 0$ for $T \rightarrow \infty$ (uncorrelatedness), (13) gives

$$
\begin{aligned}
f_{\rho_{1}, \rho_{2}}\left(\rho_{1}, \rho_{2}\right) \rightarrow & \frac{\rho_{1} \rho_{2}}{\sigma_{N}^{4}} \exp \left(-\frac{\rho_{1}^{2}+\rho_{2}^{2}+2 A_{0}^{2}}{2 \sigma_{N}^{2}}\right) \\
& \times I_{0}\left(\frac{A_{0} \rho_{1}}{\sigma_{N}^{2}}\right) I_{0}\left(\frac{A_{0} \rho_{2}}{\sigma_{N}^{2}}\right) .
\end{aligned}
$$

Since $I_{m}(x)=\frac{(x / 2)^{m}}{m !} \rightarrow \begin{cases}0 ; & m \neq 0 \\ 1, & m \neq 0,\end{cases}$

Eq. (15) can be rewritten as

$$
\begin{aligned}
f_{\rho_{1} \rho_{2}}\left(\rho_{1}, \rho_{2}\right) \underset{r \rightarrow \infty}{\rightarrow} & \frac{\rho_{1}}{\sigma_{N}^{2}} \exp \left(-\frac{\rho_{1}^{2}+A_{0}^{2}}{2 \sigma_{N}^{2}}\right) I_{0}\left(\frac{A_{0} \rho_{1}}{\sigma_{N}^{2}}\right) \frac{\rho_{2}}{\sigma_{N}^{2}} \\
& \times \exp \left(-\frac{\rho_{1}^{2}+A_{0}^{2}}{2 \sigma_{N}^{2}}\right) I_{0}\left(\frac{A_{0} \rho_{2}}{\sigma_{N}^{2}}\right),
\end{aligned}
$$

i.e., $f_{\rho_{1}, \rho_{2}}\left(\rho_{1}, \rho_{2}\right) \underset{T \rightarrow \infty}{\rightarrow} f_{\rho_{1}}\left(\rho_{1}\right) \cdot f_{\rho_{2}}\left(\rho_{2}\right)$.

We can now proceed to evaluate the one-step transition probabilities of the Markov model.

$P_{12}=a=\int_{0}^{\rho_{0}} \int_{\rho_{0}}^{\infty} f_{\rho_{1} \rho_{2}}\left(\rho_{1}, \rho_{2}\right) d \rho_{1} d \rho_{2} / \int_{\rho_{0}}^{\infty} f_{\rho}(\rho) d \rho$

$P_{21}=b=\int_{0}^{\rho_{0}} \int_{0}^{\rho_{0}} f_{\rho_{1} \rho_{2}}\left(\rho_{1}, \rho_{2}\right) d \rho_{1} d \rho_{2} / \int_{0}^{\rho_{0}} f_{\rho}(\rho) d \rho$

(and $P_{11}=1-P_{12}, P_{22}=1-P_{21}$ ).

The double integrals in (18) and (19) can be evaluated as functions of

$$
\Sigma_{1}=\int_{0}^{\rho_{1}} \int_{0}^{\rho_{0}} f_{\rho_{1} \rho_{2}}\left(\rho_{1}, \rho_{2}\right) d \rho_{1} d \rho_{2}
$$

Although $\Sigma_{1}$ is symmetric with respect to $\rho_{1}$ and $\rho_{2}$, it cannot be expressed as a product of one function of $\rho_{1}$ and one of $\rho_{2}$. Instead, we can rewrite (20), taking (13) into account, as follows:

$$
\begin{aligned}
\Sigma_{1}= & \int_{0}^{\rho_{0}} \frac{\rho_{1}}{\sigma_{N}^{4}\left(1-\rho_{0}^{2}\right)} \exp \left(-\frac{\rho_{1}^{2}}{2 \sigma_{N}^{2}\left(1-\rho_{0}^{2}\right)}\right. \\
& \left.-\frac{A_{0}^{2}}{\sigma_{N}^{2}\left(1+\rho_{0}\right)}\right) \int_{0}^{\rho_{0}} \rho_{2} \exp \left(-\frac{\rho_{2}^{2}}{2 \sigma_{N}^{2}\left(1-\rho_{0}^{2}\right)}\right) \\
& \times \sum_{m=0}^{\infty} I_{m}\left(\frac{\rho_{0} \rho_{1} \rho_{2}}{\sigma_{N}^{2}\left(1-\rho_{0}^{2}\right)}\right) \\
& \times I_{m}\left(\frac{A_{0} \rho_{1}}{\sigma_{N}^{2}\left(1+\rho_{0}^{2}\right)}\right) I_{m}\left(\frac{A_{0} \rho_{2}}{\sigma_{N}^{2}\left(1+\rho_{0}^{2}\right)}\right) d \rho_{2} d \rho_{1} .
\end{aligned}
$$

In the above, $A_{0}$ is identical to $R_{S}$ of Eq. (3).

Having evaluated $\Sigma_{1}$, Eqs. (18) $-(19)$ can be expressed as

$$
\begin{aligned}
& P_{12}=\left(P_{u}-\Sigma_{1}\right) /\left(1-P_{u}\right), \\
& P_{21}=1-\Sigma_{1} / P_{u}, \\
& P_{11}=1-P_{12}, \quad P_{22}=1-P_{21},
\end{aligned}
$$

where $P_{u}$ is the (unconditional probability of $\rho$ being less than $\rho_{0}$. Efforts to simplify the evaluation of $\Sigma_{1}$ in Eq. (21) were not successful. The double numerical integration of a function involving the infinite sum of products of three modified Bessel functions was expected to and did actually produce computational problems (excessive CPU time). These were partially alleviated using the asymptotic properties of the Bessel functions involved in determining the tolerances employed in terminating the evaluation of the infinite summations. Still, for extreme (that is, too small or too large) detection thresholds, the computational effort is unacceptably large. However, this is not expected to be a problem in practice since we do not need to use such extreme thresholds-in fact, they result in memoryless Markov models, and the problem does not exist, since the evaluation of the relevant one-step transition probabilities just requires the knowledge of the unconditional distributions.

\section{IMPLEMENTING THE MODELS}

The results obtained using our model with a variety of-fictitious but hopefully appropriate-inputs for the parameters involved, namely $\sigma_{N}^{2}, v, R_{S}$, the time step, and the detection threshold. ${ }^{12}$

Figures 2-3 present typical results using $\sigma_{N}^{2}=1.58, R_{S}$ $=2.23,\left(R_{S}^{2}=5.\right) v=0.2 \mathrm{~Hz}$, a time step of $0.4 \mathrm{~s}$, and thresholds of $\rho_{0}=1.58$. $\sigma_{N}^{2}$ and $R_{S}$ were picked from an unsaturated example,' and the rest were chosen by the authors and are more or less arbitrary. It is seen ${ }^{12}$ that, although the density of the holding time is very sensitive to the magnitude of the detection threshold, the density of the interarrival time is much less so, being almost independent of the threshold. This reminds us of what we would get in a pure sinusoidal signal situation, where we have a constant interarrival time (equal to the period of the sinusoidal signal) but different holding times for each threshold. Such a result was not observed in our previous study of the detection process 


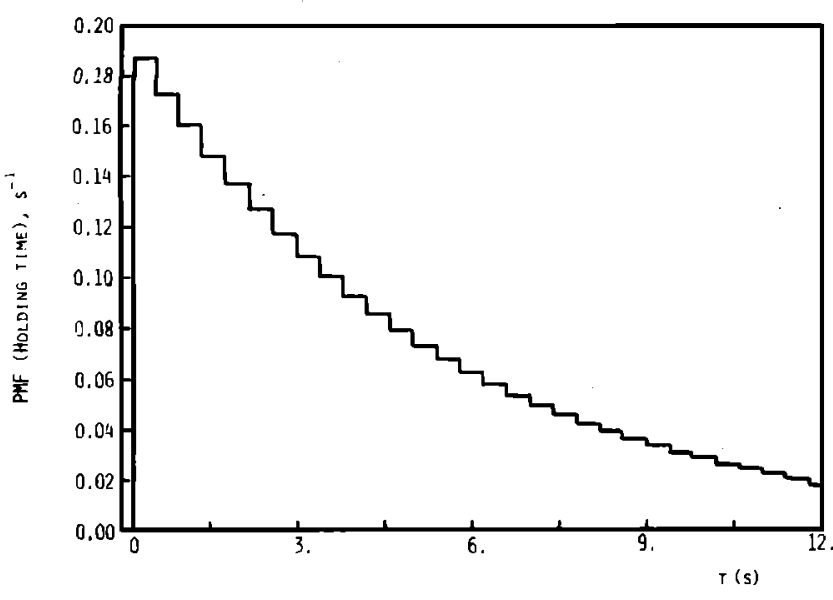

FIG. 2. Holding time, $\rho_{0}=1.58, T=0.4 \mathrm{~s}, R_{S}=2.23$.

using the phase random model for ocean propagation. ${ }^{8}$ In the unsaturated case, $\rho$ is obviously not a strict sinusoid and, hence, we do not get the above $\delta$-function densities for the interarrival and holding times. However, we also show ${ }^{12}$ that the timing of detection events (i.e., the density of the interarrival times) is almost independent of the detection threshold. This threshold makes its presence felt only in the densities of the holding time, in which we quite obviously have shorter holding times for higher thresholds. We have also obtained results ${ }^{12}$ demonstrating the relative insensitivity of the above results to changes in the time step $T$.

\section{CONCLUSIONS}

In this letter, an analytical model for the unsaturated acoustic detection process was presented, and probability mass functions for the interarrival and holding times were

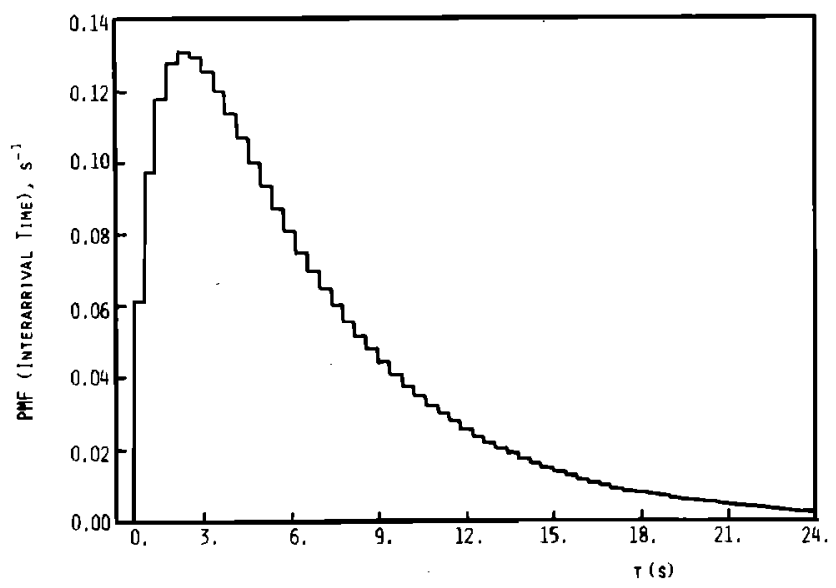

FIG. 3. Interarrival time, $\rho_{0}=1.58, T=0.4 \mathrm{~s}, R_{S}=2.23$. derived. The unsaturated mode of acoustic propagation was seen to exhibit different characteristics than the previously developed phase random acoustic detection models. A major difference between these two modes of acoustic propagation lies in the narrower (for the unsaturated case) distribution of $\rho$, which approaches a normal density as $R_{S}$ grows large. A more striking difference lies in the relative independence of the interarrival time PMF to the detection threshold $\rho_{0}$ for the unsaturated case, a property not observed in the fully saturated models.

\section{ACKNOWLEDGMENTS}

The work has been partially supported by the Office of Naval Research, Contract No. N00014-79-C-0238. We are indebted to Dr. Peter Mikhalevsky, U. S. Navy, for providing us with the first-order statistics for the unsaturated process and for his comments.

'P. N. Mikhalevsky, "Envelope Statistics of Partially Saturated Processes," J. Acoust. Soc. Am. 72, 151-158 (1982).

${ }^{2}$ S. O. Rice, “Mathematical Analysis of Random Noise," Bell Syst. Tech. J. 23 (1944); and 24 (1945).

${ }^{3}$ W. R. Hamblen, "Phase Random Multipath Model for Acoustic Signal Fluctuations in the Ocean and its Comparison with Data," Ph.D. thesis, MIT, 1977.

4I. Dyer, "Statistics of Sound Propagation in the Ocean," J. Acoust. Soc. Am. 48, 337-345 (1970).

${ }^{5}$ I. Dyer, "Statistics of Distant Shipping Noise," J. Acoust. Soc. Am. 53, 564 (1973).

${ }^{6} \mathrm{P}$. N. Mikhalevsky, "First Order Statistics for Finite Bandwidth Multipath Signals with and without Frequency or Phase Modulation," J. Acoust. Soc. Am. 66, 751-762 (1979).

${ }^{7}$ H. N. Psaraftis, A. N. Perakis, and P. N. Mikhalevsky, "New Models for the Ocean Acoustic Detection Process," J. Acoust. Soc. Am. 69, 17241734 (1981).

${ }^{8}$ H. N. Psaraftis, A. N. Perakis, and P. N. Mikhalevsky, "Memory Detection Models for Phase-Random Ocean Acoustic Fluctuations," Proceedings, International Conference on Communications, Denver, $\mathrm{CO}$, June 1981, pp. 12.6.1-12.6.5.

${ }^{9}$ A. N. Perakis, H. N. Psaraftis, and H. I. Gonzalez, "Detection Modeling of Unsaturated and Partially Saturated Acoustic Signals," in Proceedings, Sixth MIT /ONR Workshop on Command and Control (MIT, Cambridge, MA, July 1983).

IOS. A. Rappaport, "Computing Approximations to the Generalized $Q$ Function and its Complement," Correspondence, IEEE Trans. Inf. Theory, 497-498 (July 1971).

"D. Middleton, An Introduction to Statistical Communication Theory (McGraw-Hill, New York, 1960).

${ }^{12}$ A. N. Perakis and H. N. Psaraftis, "Discrete-Time Detection Modeling for Unsaturated Ocean Propagation," Working Paper No. OE-ONR-822, October 1982. 